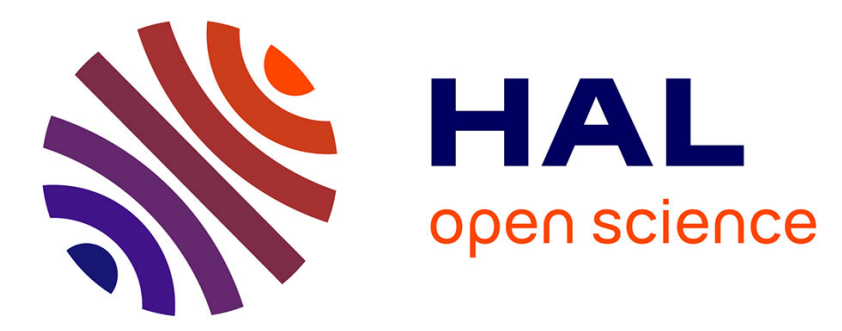

\title{
Influence of static disorder and polaronic band formation on the interfacial electron transfer in organic photovoltaic devices
}

Kevin-Davis Richler, Didier Mayou

\section{- To cite this version:}

Kevin-Davis Richler, Didier Mayou. Influence of static disorder and polaronic band formation on the interfacial electron transfer in organic photovoltaic devices. Physical Review B, 2019, 99 (19), pp.195151. 10.1103/PhysRevB.99.195151 . hal-02400983

\section{HAL Id: hal-02400983 https://hal.science/hal-02400983}

Submitted on 9 Dec 2019

HAL is a multi-disciplinary open access archive for the deposit and dissemination of scientific research documents, whether they are published or not. The documents may come from teaching and research institutions in France or abroad, or from public or private research centers.
L'archive ouverte pluridisciplinaire $\mathbf{H A L}$, est destinée au dépôt et à la diffusion de documents scientifiques de niveau recherche, publiés ou non, émanant des établissements d'enseignement et de recherche français ou étrangers, des laboratoires publics ou privés. 


\title{
Influence of static disorder and polaronic band formation on the interfacial electron transfer in organic photovoltaic devices
}

\author{
Kevin-Davis Richler and Didier Mayou \\ University Grenoble Alpes, Institut NEEL, F-38042 Grenoble, France \\ and CNRS, Institut NEEL, F-38042 Grenoble, France
}

(Received 11 February 2019; revised manuscript received 8 April 2019; published 28 May 2019)

\begin{abstract}
Understanding the interfacial charge separation mechanism in organic photovoltaics requires, due to its high level of complexity, bridging between chemistry and physics. To elucidate the charge separation mechanism, we present a fully quantum-dynamical simulation of a generic one-dimensional Hamiltonian, whose physical parameters model prototypical Phenyl- $\mathrm{C}_{61}$-butyric acid methyl ester or $C_{60}$ acceptor systems. We then provide microscopic evidence of the influence random static and dynamic potentials have on the interfacial chargeinjection rate. In particular, we unveil that dynamic potentials, due to strong electron-vibration interactions, can lead to the formation of polaronic bands. Such dynamical potentials, when compared to random static potentials, can provide the main detrimental influence on the efficiency of the process of interfacial charge separation.
\end{abstract}

DOI: 10.1103/PhysRevB.99.195151

\section{INTRODUCTION}

Organic photovoltaic devices are currently attracting intense interest due to their potentially cheap production and their ability for environmentally friendly power generation [1-3]. The efficiency of these organic photovoltaic (OPV) devices strongly depends on the charge separation process between materials that transport electrons (usually a fullerene derivative) or holes (usually a polymer). The energy offset caused by this interface drives electrons from the donor to the acceptor, while leaving holes behind. Once the charges are in separate phases, they need to overcome their mutual Coulomb attraction. This binding energy is in the range of 0.1 to $0.5 \mathrm{eV}$ [4-6], which is much larger than the thermal energy of about $25 \mathrm{meV}$ at room temperature. Surprisingly, the charge separation process and, eventually, the formation of free charges that can be extracted at the electrodes are still very efficient $[7,8]$. In particular, experimental studies have shown that the charge separation occurs on ultrafast timescales in the 10 to $100 \mathrm{fs}$ range [9-11]. At present, the mechanism responsible for charge separation is not well understood and is still actively debated.

An obvious question in organic materials relates to the relative importance of static and dynamic potentials and their effect on the charge separation process. The static (timeindependent) potential reflects the electron-hole Coulomb interaction and the spatial disorder caused by electrostatic interactions resulting from the different environments in which each molecule is placed. This is particularly true for organic devices made up of two different disordered materials $[12,13]$ where the spatial disorder usually originates from the rather large permanent electric dipole of Phenyl- $\mathrm{C}_{61}$-butyric acid methyl ester (PCBM). However, we stress here, that in contrast to PCBM, molecular dynamic simulations have shown that $C_{60}$, which has no permanent dipole, exhibits extremely limited disorder, which thus can, to a good extent, be neglected $[14,15]$. On the contrary, the dynamic potential is related to electron-vibration interactions that result in a time-dependent variation of microscopic transport parameters. Although debated [16,17], there are indications that, for charge separation, this effect could contribute $[18,19]$. Thus, the consideration of static and dynamic potentials seems unavoidable for a fully microscopic understanding of the charge separation mechanism in OPV devices. However, such microscopic descriptions are computationally challenging. To tackle these challenges, various numerical methods such as exact diagonalization [20,21], diagrammatic Monte Carlo [22,23], time-dependent density functional theory [24], and other approaches $[25,26]$ have been proposed. However, either these methods are quite expensive from a computational point of view, or their application to nontranslationally invariant systems remains unclear.

To overcome these difficulties the inhomogeneous version of the dynamical mean-field theory approximation (I-DMFT) [27-33], a powerful nonperturbative technique for strongly interacting systems, has been introduced. By applying the I-DMFT approximation to a generic one-dimensional model Hamiltonian, whose parameters model prototypical PCBM and $C_{60}$-based acceptor systems, we provide a fully quantumdynamical simulation of the charge separation process taking static (disorder plus electron-hole Coulomb interaction) and dynamic potentials (electron-vibration interaction) into consideration. This provides the possibility to compute the charge injection rate at the donor-acceptor interface. Our work provides a step forward to a long-standing challenge in $\mathrm{OPV}$, thereby bridging chemistry and physics. In particular we unveil here that dynamic potentials (related to polaron formation), when compared to random static potentials, present the main detrimental loss mechanism in OPV devices. Yet dynamical potentials can, in some instances, lead to an enhancement of the charge transfer process.

This paper is organized as follows. Section II is divided into two parts: In Sec. II A we introduce the generic 
one-dimensional Holstein-based Hamiltonian to model the charge carrier dynamics across organic model interfaces. In Sec. II B we briefly introduce the used I-DMFT approximation and comment on its general numerical aspects. In Sec. III we apply the I-DMFT approximation to study the charge carrier dynamics across donor-acceptor model interfaces. Finally, we provide a brief conclusion and outlook in Sec. IV.

\section{METHODOLOGY}

Inspired by the Holstein model used in [34], we use the following generic one-dimensional Hamiltonian to describe the microscopic charge-transfer process of an electron at the molecular donor-acceptor interface:

$$
\begin{aligned}
H= & \epsilon_{0} c_{0}^{+} c_{0}+m c_{0}^{+} c_{1}+m c_{1}^{+} c_{0}+\sum_{i=1} \epsilon_{i} c_{i}^{+} c_{i} \\
& -\sum_{i=1} \frac{V}{i} c_{i}^{+} c_{i}+\sum_{i=1} J\left(c_{i}^{+} c_{i+1}+c_{i}^{+} c_{i-1}\right) \\
& +\sum_{i=1} \Omega a_{i}^{+} a_{i}+\sum_{i=1} g c_{i}^{+} c_{i}\left(a_{i}^{+}+a_{i}\right),
\end{aligned}
$$

where $c_{i}^{+}\left(c_{i}\right)$ is the creation (annihilation) operator of electrons, $a_{i}^{+}\left(a_{i}\right)$ is the creation (annihilation) operator of phonons, $\Omega$ is the relevant phonon frequency ( $\hbar=1$ throughout this work), $g$ is the electron-phonon coupling strength, $m$ is the tunneling amplitude between the lowest unoccupied molecular orbital (LUMO) of the donor (site $i=0$ ) and the first site of the LUMO of the acceptor, $V$ determines the Coulomb potential, $J$ is the electron hopping parameter, $\epsilon_{0}$ is the energy of the incoming electron, and $\epsilon_{i}$ is the energy level of a molecule on the acceptor site, which is taken to be a random variable (with a mean $\epsilon$ and a standard derivation $\sigma_{\epsilon}$ ) drawn from a rectangular distribution. In this model, the static potential is given explicitly by the distribution of the onsite energies $\epsilon_{i}$ and the Coulomb potential $V$ between the electron and hole, while the dynamic potential is caused by the electron-phonon coupling term $\sum_{i} g c_{i}^{+} c_{i}\left(a_{i}^{+}+a_{i}\right)$.

At this point, we want to note that we make the following assumptions. First, we do not model the hole dynamics since the effects of hole diffusion usually lead to a reduction of only the Coulomb interaction, resulting in an increase of the electron-hole separation yield [35,36], and since hole transfer typically occurs on timescales in the 1 to 2 ps range, which is several orders of magnitude slower than electron transfer [37]. Second, we use a one-dimensional model to describe a complex three-dimensional bulk molecular heterostructure since the main features of the Holstein polaron do not depend strongly on the dimensionality of the system [38] and since we expect that the realistic, three-dimensional nature of the system will lead to mainly quantitative changes in the quantum efficiency. Third, we assume only a single high-frequency intramolecular mode of vibration (on the order of $1600 \mathrm{~cm}^{-1}$, i.e., a period of roughly $20 \mathrm{fs}$ ), although the involvement of multiple phonon modes provides additional transfer channels. However, in this work, we are interested in electron dynamics that concern the fast electron unbinding from the Coulomb well, which occurs on timescales of 10 to $100 \mathrm{fs}$. Therefore, the impact of weakly coupled low-frequency modes of vibra- tion (on the order of $\lesssim 80 \mathrm{~cm}^{-1}$, i.e., a period of roughly $415 \mathrm{fs}$ ) can be neglected since final equilibration occurs at longer relaxation times [39]. Fourth, all computations are presented at zero temperature, taking no entropic effects into consideration, since electronic and vibrational energy scales are much larger than $k_{\mathrm{B}} T$, where $k_{\mathrm{B}}$ is Boltzmann's constant and $T$ is the temperature [40], and since we expect that a change in entropy plays a diminished role in the charge separation process in one-dimensional systems [41]. The driving force for the charge separation mechanism is thus entirely of a quantummechanical nature stemming from the coupling of an initially discrete state (electron at the interface) to a final state in the continuum (electron on the acceptor side surrounded by a cloud of phonon excitations). Fifth, we note that in the absence of electron-phonon interaction, scattering on defects can lead to Anderson localization [42], and it will hinder electron transfer across the interface. Since it is well known that in the case of one-dimensional infinite disordered systems, any amount of disorder produces Anderson localization, we have embedded the system into an effective medium which has been computed using the coherent potential approximation (CPA). The absence of Anderson localization within CPA [43] will then mimic a system without localization at moderate disorder (except very close to the band edges) throughout this work.

The approach that we propose is the use of the singlepolaron I-DMFT approximation. The aim of I-DMFT is to fully address the relevant spatial variations of the physical properties while still affording a good description of the physical processes of interest. A crucial aspect of I-DMFT is that it provides an interpolation between the noninteracting case, in which it gives the exact solution of the problem, and the strong-coupling limit, in which it also becomes exact. In I-DMFT the local Green's function is for one given realization of disorder and is defined as

$$
G_{i i}(z)^{-1}=z-\epsilon_{i}-\Delta_{i}(z)-\Sigma_{i}(z)
$$

where $\Delta_{i}(z)$ and $\Sigma_{i}(z)$ are the hybridization function and the self-energy at site $i$, respectively, and $z=E+i \eta$, with $\eta$ being an infinitesimally small number. In standard I-DMFT procedures a self-consistent solution is obtained iteratively at each $z$ where one needs to compute repeatedly the diagonal of the inverse of a complex matrix whose dimension equals the number of lattice sites. Using conventional linear-algebra algorithms, this problem becomes cubic with the size of the system [44].

We use an alternative approach to solve the I-DMFT selfconsistency equations which is based on Haydock's recursion scheme applied to suitably defined Hamiltonians and, in particular, which does not require the inverse of a complex matrix. Instead, the Hamiltonian (1) is solved on the full lattice under the approximation that the electron-phonon self-energy is local depending on the frequency of only the local phonon. Self-consistency equations are then expressed in Hilbert space such that the recursion technique by Haydock [45] can be used, which makes this method immediately generalizable to any lattice geometry and/or disorder distribution while easily handling inhomogeneous systems of up to $10^{3}$ lattice sites in less than $24 \mathrm{~h}$ (sequential computation on an Intel 


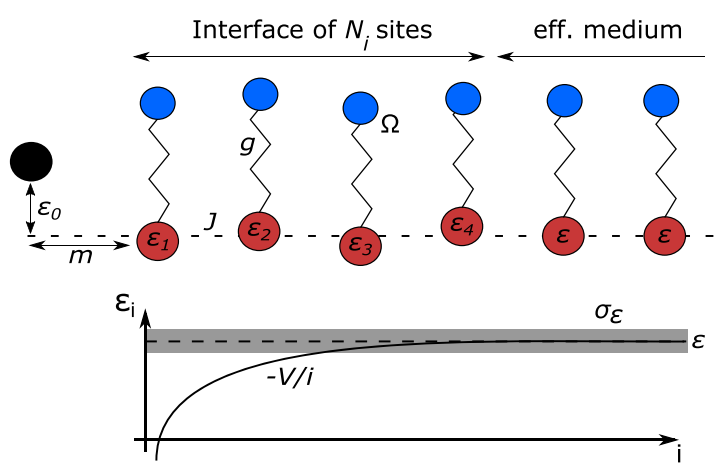

FIG. 1. Schematic representation of the Hamiltonian (1) describing the electron dynamics at the donor-acceptor interface. The black curve represents the Coulomb attraction from the hole (black site), and the dashed area represents the spread around the mean energy value $\epsilon$. We embedded the leftmost nonequivalent site $N_{i}$ into an effective medium which was computed using the coherent potential approximation.

Xeon E5-2670 processor). A detailed explanation of the used I-DMFT approach can be found in [46].

Finally, let us comment on general numerical aspects of the proposed I-DMFT formalism. The number of possible phonon configurations is infinite but can be restricted to a finite, sufficiently large number in actual calculations by choosing $M \gg g^{2} / \Omega^{2}=\alpha^{2}$, where $M$ is the maximum number of phonon excitations per site. Further, we simulate only a finite part of the total size of the system $N$. We then embed the leftmost site into an effective medium which has been computed using the CPA. We thus define a number $N_{i}<N$ of nonequivalent lattice sites along the surface. Choosing $N=2000, M=60$, and $N_{i}=300$ ensures that all results are independent of any system size characteristics while keeping modest computational complexity. A pictorial representation of the proposed model is depicted in Fig. 1.

\section{RESULTS}

In the following we express all energies in units of $J$ (energy unit is $J \approx 0.2 \mathrm{eV}$ and time unit is $1 / J \approx 3.3 \mathrm{fs}$ ) and take $m=0.5, V=1.5, \Omega=1.0, \epsilon=0.0$, and $\sigma_{\epsilon}=0.0,0.1$ while keeping $g$ and $\epsilon_{0}$ as independent parameters. All are chosen such that they are in a realistic experimental range to model the prototypical PCMB $\left(\sigma_{\epsilon}=0.1\right)$ and $C_{60}\left(\sigma_{\epsilon}=0.0\right)$ acceptor systems [47-51]. We have checked that all results are qualitatively insensitive to different disorder configurations, a result that has been found in all tested cases throughout this work. Finally, we note that throughout this paper our initial state at time $t=0$ will consist of an electron at site $i=0$ having energy $\epsilon_{0}$ and no phonon modes excited, i.e., $|\psi(t=0)\rangle=c_{0}^{+}|0\rangle$, with $|0\rangle$ being the vacuum state for phonons and electrons.

First, we present in Fig. 2 the probability density of the wave function $\left|\mathcal{K}_{\gamma}\right\rangle$ on the acceptor

$$
n_{\gamma}(t)=\left|\left\langle\mathcal{K}_{\gamma} \mid \psi(t)\right\rangle\right|^{2}
$$

where $\left|\mathcal{K}_{\gamma}\right\rangle$ is the orthonormal basis vector of the $N$ dimensional Krylov space $\mathrm{KS}_{N}$ [52]. The Krylov states $\left|\mathcal{K}_{\gamma}\right\rangle$, which are computed by a Lanczos-based recursion method [45], represent a basis of excitations of the many-body system (electron and phonon modes) that progressively spread away from the interface into the acceptor. The time evolution of the wave function in Krylov space is then defined as $|\psi(t)\rangle=$ $e^{-i \mathcal{H}_{\mathrm{KS}_{N} t}}\left|\mathcal{K}_{0}\right\rangle$, with $\mathcal{H}_{\mathrm{KS}_{N}}$ being the reduced Hamiltonian of the Krylov subspace $\mathrm{KS}_{N}$. The time evolution is then determined by an exact diagonalization technique by choosing the first Krylov-space vector equal to the initial state, i.e., $\left|\mathcal{K}_{0}\right\rangle=$ $c_{0}^{+}|0\rangle$ (see the Appendix for a brief introduction to the Krylov subspace method and computation of wave function $\left|\mathcal{K}_{\gamma}\right\rangle$ ). This provides an efficient way to extract the essential character of the Hamiltonian (1) while using a limited number of basis sets. We stress here that the size of the system (2000 states in the Krylov space) is sufficient to prevent the wave packet from bouncing back at the boundary.

We find, in the limit of sole dynamic potentials $\left(\alpha^{2}=0.5\right.$, $\sigma_{\epsilon}=0.0$ ) shown in Fig. 2(a), that the probability weight near the interface progressively decays with time and the particle is fully delocalized. Upon increasing the electron-phonon interaction $\left(\alpha^{2}=1.5, \sigma_{\epsilon}=0.0\right)$, the local density of states (LDOS) fragments into polaronic subbands (self-trapping) (a)

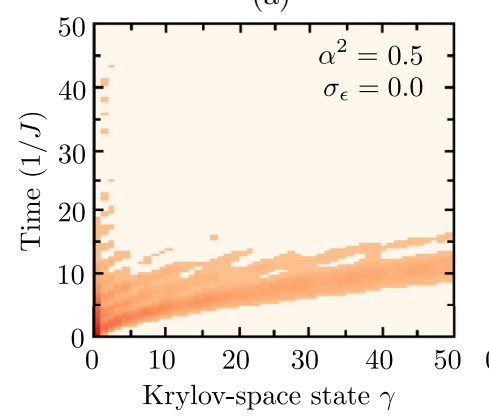

(b)

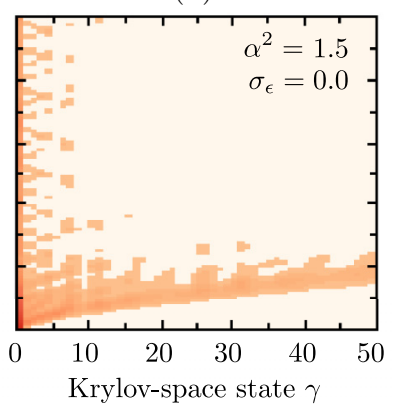

(c)

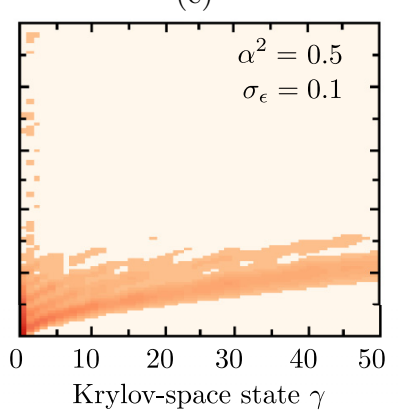

(d)

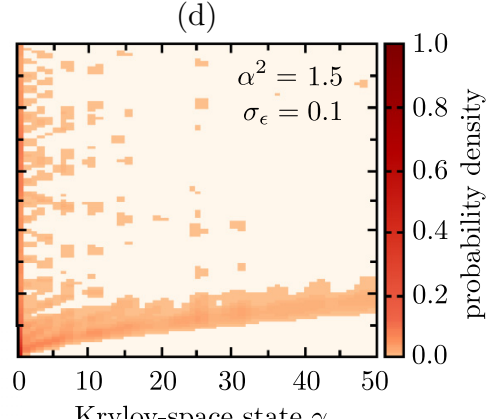

FIG. 2. Time evolution of the probability density $n_{\gamma}(t)$ at each state $\gamma$ in Krylov space, where all calculations are shown for an incoming electron energy $\epsilon_{0}=-0.5$ that is taken in the band of delocalized states (time unit is $1 / J \approx 3.3 \mathrm{fs}$ ). (a) and (b) are only for dynamic potentials, while (c) and (d) are for dynamic potentials and static disorder. In fact, molecular dynamics simulations have shown that contrary to PCBM fullerenes, $C_{60}$ exhibits extremely limited disorder in its crystalline phase, which can thus, to a good extent, be neglected [14,15]. (b) and (d) show a localization effect near the interface, which is, however, superposed with outgoing wave packets, resulting in a poor interfacial charge-transfer efficiency. We did not attempt to estimate the numerical error, but it should be of the order of the fluctuations visible in the plot. 
where the strongly renormalized width of the polaronic subbands arises as the new energy scale [53]. As can be seen in Fig. 2(b), self-trapping of the electron hinders, in this case, the interfacial electron transfer drastically as parts of the wave function remain localized at the interface, resulting in a poor but finite interfacial charge-transfer efficiency. In Figs. 2(c) and 2(d) we present the combined effect of static spatial disorder and weak $\left(\alpha^{2}=0.5, \sigma_{\epsilon}=0.1\right)$ and strong $\left(\alpha^{2}=1.5, \sigma_{\epsilon}=0.1\right)$ dynamic potentials, respectively. As can be seen, the physical pictures in Figs. 2(c) and 2(d) do not change quantitatively when compared to Figs. 2(a) and 2(b), respectively, an outcome we find throughout this work.

Before proceeding we stress here that the LDOS is a key factor since its shape and, in particular, the energy distribution of electronic states are found to determine the value of practically achievable injection energies. This comes in handy when using the proposed formalism [46]. In particular, we have found that taking the incoming electron energy outside the band of delocalized states completely suppresses, as expected, interfacial charge transfer (an electron becomes localized at the interface) while the incoming electron energy that is taken in the band of delocalized states results in interfacial charge transfer.

By making use of the I-DMFT-computed Green's function $G_{11}(z)$ and by applying Fermi's golden rule we now quantify, as a function of the incoming electron energy $\epsilon_{0}$, the quantummechanical donor-acceptor interface transfer rate,

$$
k_{I n j}\left(\epsilon_{0}\right)=-2 m^{2} \operatorname{Im} G_{11}\left(\epsilon_{0}+i \eta\right),
$$

where $\operatorname{Im} G_{11}(z)$ is the imaginary part of the Green's function $G_{11}(z)$. This presents a simple measure of the efficiency for charge transfer at the donor-acceptor interface, but note the transfer rate is related, but not equal, to the internal quantum efficiency, which is commonly extracted from experiments. The interface transfer rate is graphically depicted in Fig. 3 for incoming electron energies of $-2 J \leqslant \epsilon_{0} \leqslant 2 J$, where this restriction is sufficient to ensure the applicability of Eq. (4) (weak coupling to a quasicontinuum of states). Although we do not model the competing electron-hole and/or geminate recombination processes, we also indicate in Fig. 3 the range of the experimentally observed recombination rate $k_{\operatorname{Rec}}$ of the order of $0.01^{-1}$ to $0.05 \mathrm{fs}^{-1}$ [9-11]. Therefore, efficient charge transfer from the donor to the acceptor occurs when the interface transfer rate $k_{I n j}$ is large compared to the competing process of charge recombination $k_{\mathrm{Rec}}$. We start considering the case of only sole, but weak, dynamic potentials $\left(\alpha^{2}=\right.$ $\left.0.5, \sigma_{\epsilon}=0.0\right)$. We find that the interfacial transfer rate is essentially, with the exception of values of $\epsilon_{0}$ close to the low-energy edge, higher than the upper experimental recombination rate for all negative values of $\epsilon_{0}$. On the contrary, this changes drastically for all positive values of $\epsilon_{0}$, where interfacial charge transfer is large only when compared to extremely low experimental ranges of recombination rates. This stems from strong relaxation processes (phonon emission) caused by molecular vibrations which are facilitated at higher energies. Upon increasing the electron-phonon interaction $\left(\alpha^{2}=1.5\right)$ multiple gaps throughout the spectrum arise, and thus multiple values of $k_{I n j}$ occur that are below the experimental value of $k_{\mathrm{Rec}}$. This is readily explained since upon increasing the electron-phonon interaction the LDOS
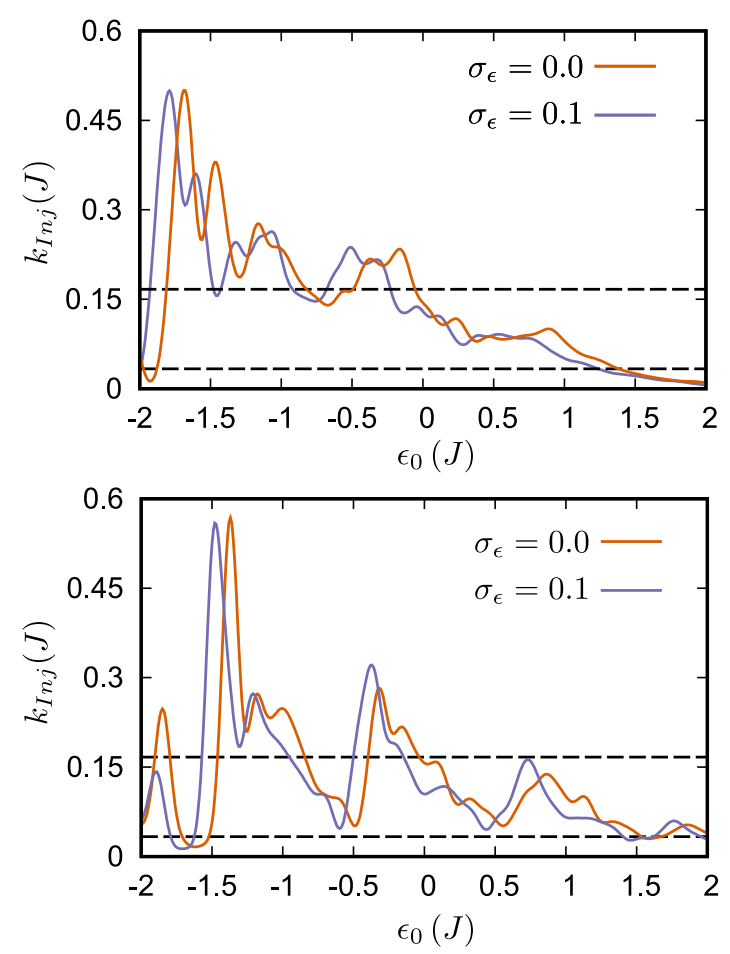

FIG. 3. We present the interface transfer rate $k_{I n j}$ (rate unit is $J \approx 0.3 \mathrm{fs}^{-1}$ ) as a function of the incoming electron energy $\epsilon_{0}$ for $\alpha^{2}=0.5,1.5$ (top to bottom). The dashed lines mark the range of experimentally observed recombination rates $k_{\operatorname{Rec}}$ of the order of $0.033 J\left(0.01 \mathrm{fs}^{-1}\right)$ to $0.167 J\left(0.05 \mathrm{fs}^{-1}\right)$ [9-11]. Transfer rates $k_{I n j}$ below $k_{\text {Rec }}$ will eventually lead to electron-hole and/or geminate recombination at the donor-acceptor interface. The bottom panel shows that by increasing the electron-phonon interaction $\alpha$, narrow polaronic subbands separated by $\Omega$ arise, an effect which hinders the range of suitable incoming electron energies $\epsilon_{0}$. We note that we did not attempt to estimate the numerical error, but it should be of the order of $\eta / J=5.0 \times 10^{-2}$ due to Gaussian broadening.

fragments into subbands that are separated by multiples of $\Omega$. This effect will drastically hinder the range of suitable incoming electron energies since the incoming electron energy $\epsilon_{0}$ must be identical to that of the allowed unoccupied state of the acceptor material. Surprisingly, this picture does not change qualitatively when considering the combined effect of static disorder and dynamic potentials. In particular, we have found that weak but static disorder does not change drastically the interfacial transfer rate for incoming electron energies of $-2 J \leqslant \epsilon_{0} \leqslant 2 J$. Finally, we note that these results are in agreement with the analysis of the time evolution of the electron density shown above.

We note here that we confine our analysis of the interfacial transfer rate by choosing realistic experimental values for all parameters. However, introducing $m \rightarrow \mu m, J \rightarrow \lambda J$ enables the scale transformation of the interfacial transfer rate $k_{I n j} \rightarrow$ $k_{\text {Inj }}^{\prime}$ into

$$
k_{\text {Inj }}^{\prime}\left(\epsilon_{0}\right)=\left(\mu^{2} / \lambda\right) k_{I n j}\left(\epsilon_{0} / \lambda\right),
$$

where $\mu$ and $\lambda$ are the scaling constants of $m$ and $J$, respectively. Through this scale transformation the results presented in Fig. 3 can then be adapted to describe physically equivalent 
systems with a different scale $k_{I n j}^{\prime}$ and set of parameters $\left(\Omega^{\prime}=\right.$ $\left.\lambda \Omega, V^{\prime}=\lambda V, g^{\prime}=\lambda g, \sigma_{\epsilon}^{\prime}=\lambda \sigma_{\epsilon}\right)$.

Finally, we note the following: First, we have found that, contrary to common OPV design suggestions $[1,2]$ in which the molecular energy level of the LUMO of the donor is aligned above the LUMO of the acceptor, charge transfer is more efficient when the opposite molecular energy level alignment occurs, i.e., when the energy level of the LUMO of the acceptor is above the energy level of the LUMO of the donor. Second, we have not found interfacial electron transfer in the limit of extremely strong electron-phonon coupling $\left(\alpha^{2} \gtrsim 8.0\right)$. This is readily explained since in this limit the coupling between the donor and the first site of the acceptor becomes much larger than the renormalized bandwidth of the polaronic subbands. Thus, one is, to a good extent, left with two eigenstates separated by a larger energy offset, giving rise to charge localization at the interface.

Third, we note that the results of our time-dependent study present some similarity to the Dirac-Frenkel time-dependent simulations in one dimension [34], which focused on the interplay between electron-vibration interaction and the interfacial Coulomb interaction between the hole and electron. Yet [34] did not take spatial disorder into consideration and focused on the quantum yield in the absence of any electronhole recombination. The quantum yield, in the absence of recombination, then simply measures the charge injected at an infinite distance from the interface; that is, the quantum yield is simply $1-P$, with $P$ being the weight of the wave function localized near the interface. Instead, our study quantifies the charge injection rate, which has more fundamental relevance since this quantity can be compared to the experimentally observed recombination rate. The quantum yield, in the presence of recombination, can then be obtained by

$$
Y \simeq \frac{k_{I n j}}{k_{I n j}+k_{\mathrm{Rec}}} .
$$

A high quantum yield is then given when $k_{I n j} \gg k_{\text {Rec }}$ holds. Moreover, the applicability of the simulation presented in Ref. [34] is limited since the approximated treatment of the many-body nature of the polaronic state (momentum average approximation) is valid only in the small bandwidth limit. In contrast to Ref. [34], the proposed I-DMFT approach is accurate over the entire parameter space [46].

\section{CONCLUSION}

To summarize, we have applied the I-DMFT approximation to a generic one-dimensional model Hamiltonian whose parameters model the charge carrier dynamics in prototypical PCBM and $C_{60}$ acceptor systems. Our results show that polaronic bands, when compared to spatial disorder, can provide the main detrimental influence on the efficiency of charge transfer of electrons across organic interfaces. From this perspective, organic molecules with moderate reorganization energies should be used preferentially in next-generation materials since increasing the electron-phonon interaction hinders the range of suitable incoming electron energies due to the fragmentation of the local density of states into narrow polaronic subbands. Interestingly, contrary to common OPV design suggestions [1,2], where the molecular energy level of the LUMO of the donor is aligned above the LUMO of the acceptor, we have found that charge transfer is more efficient when the opposite molecular energy level alignment occurs, i.e., when the energy level of the LUMO of the acceptor is above the energy level of the LUMO of the donor. Finally, we emphasize here that the easy numerical implementation of the I-DMFT approximation [46] allows one to study a variety of recently proposed and perhaps more realistic donor-acceptor model systems. In particular, I-DMFT enables us to investigate the impact of electric fields induced by energy level pinning [54], structural heterogeneity as a function of distance to the interface $[55,56]$, and gradients in the energy landscape [57,58]. These problems were previously difficult to access but may help the charge separation process drastically. Open questions we reserve for future work.

\section{ACKNOWLEDGMENTS}

The authors thank S. Fratini, S. Ciuchi, and G. D'Avino for stimulating discussions. K.-D. Richler acknowledges the LANEF framework (ANR-10-LABX-0051) for its support with mutualized infrastructure.

\section{APPENDIX: KRYLOV SUBSPACE METHOD}

We recall here a general definition of the Krylov subspace method used in this work. The Krylov subspace of dimension $N\left(\mathrm{KS}_{N}\right)$ [52] is the linear subspace spanned by

$$
\mathrm{KS}_{N}\left(\left|\phi_{0}\right\rangle\right)=\operatorname{span}\left\{\left|\phi_{0}\right\rangle, H\left|\phi_{0}\right\rangle, \ldots, H^{N-1}\left|\phi_{0}\right\rangle\right\} .
$$

Here $\left|\phi_{0}\right\rangle$ represents a suitably chosen vector of the original Hilbert space, and we will denote in the following the orthonormalized basis vectors in $\mathrm{KS}_{N}$ by

$$
\left|\mathcal{K}_{0}\right\rangle,\left|\mathcal{K}_{1}\right\rangle,\left|\mathcal{K}_{2}\right\rangle, \ldots,\left|\mathcal{K}_{N-1}\right\rangle \equiv \mathrm{KS}_{N},
$$

with $\left|\mathcal{K}_{0}\right\rangle=\left|\phi_{0}\right\rangle$ being defined as the initially chosen wave function. An orthogonal basis of $\mathrm{KS}_{N}$ can then be constructed with the method of Lanczos [59], an iterative procedure that is capable of constructing the Krylov space via a three-term recurrence relation:

$$
H\left|\mathcal{K}_{n}\right\rangle=a(n)\left|\mathcal{K}_{n}\right\rangle+b(n)\left|\mathcal{K}_{n+1}\right\rangle+b(n-1)\left|\mathcal{K}_{n-1}\right\rangle,
$$

with the initial conditions $\left|\mathcal{K}_{0}\right\rangle=0, b(-1)=0$ and where $\left|\mathcal{K}_{n}\right\rangle$ obeys the orthogonality relation $\left\langle\mathcal{K}_{n} \mid \mathcal{K}_{m}\right\rangle=\delta_{n, m}$. The reduced Hamiltonian matrix $H_{\mathcal{K} \mathcal{S}_{\mathcal{N}}}$ in $\mathrm{KS}_{N}$ then reads

$$
H_{\mathcal{K S}}=\left[\begin{array}{cccccc}
a(0) & b(0) & 0 & 0 & \ldots & \\
b(0) & a(1) & b(1) & 0 & 0 & \ldots \\
0 & b(1) & a(2) & b(2) & 0 & \ldots \\
\vdots & \ddots & \ddots & \ddots & \ddots & \ddots
\end{array}\right] .
$$

The time evolution of the wave function $\left|\mathcal{K}_{0}\right\rangle$ in the Krylov subspace $\mathrm{KS}_{N}$ is then defined by

$$
\begin{aligned}
|\psi(t)\rangle & =e^{-i H_{\mathrm{KS}_{\mathcal{N}}} t}\left|\mathcal{K}_{0}\right\rangle \\
& =\sum_{l=0}^{N} e^{-i E_{\mathrm{KS}_{\mathcal{N}}}(l) t}\left\langle Z_{\mathrm{KS}_{\mathcal{N}}}(l) \mid \mathcal{K}_{0}\right\rangle\left|Z_{\mathrm{KS}_{\mathcal{N}}}(l)\right\rangle .
\end{aligned}
$$

Here $E_{\mathrm{KS}_{\mathcal{N}}}(l)$ and $\left|Z_{\mathrm{KS}_{\mathcal{N}}}(l)\right\rangle$ represent the $l$ th eigenvalue and eigenvector of the Hamiltonian $H_{\mathrm{KS}_{\mathcal{N}}}$, respectively, which 


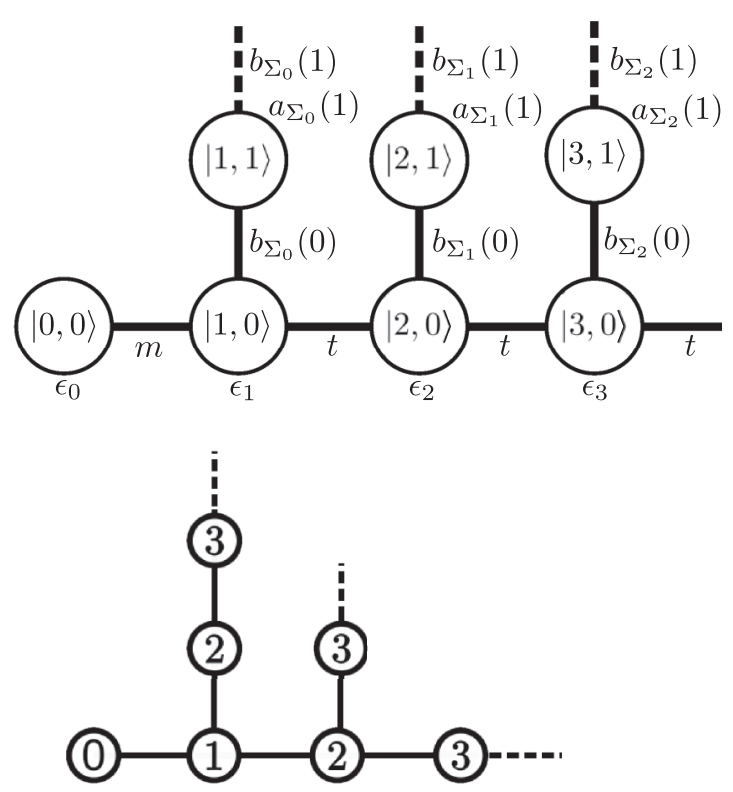

FIG. 4. Top: tight-binding representation of the Hamiltonian $H_{I D M F T}$, where $H_{I D M F T}$ and, especially, the coefficients $a_{\Sigma_{n}}(n)$ and $b_{\Sigma_{n}}(n)$ were computed using the I-DMFT formalism presented in Ref. [46]. Bottom: the most outward extension of the Krylov subspace vector $\left|\mathcal{K}_{N}\right\rangle$ for the first three steps of the recursion scheme.

have been determined by exact diagonalization (due to the greatly reduced size of the Hamiltonian matrix $H_{\mathrm{KS}_{\mathcal{N}}}$ ).

To further illustrate the extension of the Krylov subspace vector $\left|\mathcal{K}_{N}\right\rangle$, we add in Fig. 4 the tight-binding representation of the Hamiltonian $H_{I D M F T}$, which was computed with the I-DMFT method presented in Ref. [46] and has been used to determine all $\left|\mathcal{K}_{N}\right\rangle$. Starting Haydock's recursion from the initial state $\left|\mathcal{K}_{0}\right\rangle=\left|\phi_{0}\right\rangle=|0,0\rangle$ (the electron at site $i=0$ has energy $\epsilon_{0}$ and no phonon modes excited), one then finds

$$
\begin{aligned}
H_{I D M F T}|0,0\rangle & =m|1,0\rangle+\epsilon_{0}|0,0\rangle \\
& =a(0)\left|\mathcal{K}_{0}\right\rangle+b(0)\left|\mathcal{K}_{1}\right\rangle .
\end{aligned}
$$

Here states in the energy-independent tight-binding representation of $H_{I D M F T}$ are labeled $|x, n\rangle$, with $x$ being the lattice coordinate and $n$ being the phonon number (see Fig. 4). Projecting on Eq. (A6) with $\left|\mathcal{K}_{0}\right\rangle$ and $\left|\mathcal{K}_{1}\right\rangle$, one then finds $a(0)=$ $\epsilon_{0}, b(0)=m$, and $\left|\mathcal{K}_{1}\right\rangle=|1,0\rangle$. In the second recursion step one then finds

$$
\begin{aligned}
H_{I D M F T}\left|\mathcal{K}_{1}\right\rangle= & t|2,0\rangle+\epsilon_{1}|1,0\rangle \\
& +b_{\Sigma_{0}}(0)|1,1\rangle+m|0,0\rangle \\
= & a(1)\left|\mathcal{K}_{1}\right\rangle+b(1)\left|\mathcal{K}_{2}\right\rangle+b(0)\left|\mathcal{K}_{0}\right\rangle
\end{aligned}
$$

The set of new recursion coefficients then is $a(1)=\epsilon_{1}, b(1)=$ $\sqrt{t^{2}+b_{\Sigma_{0}}^{2}(0)}$, and the new wave function reads

$$
\left|\mathcal{K}_{2}\right\rangle=\frac{t|2,0\rangle}{b(1)}+\frac{b_{\Sigma_{0}}(0)|1,1\rangle}{b(1)} .
$$

This iterative three-term recurrence procedure is repeated until all recursion coefficients $a(n), b(n)$ and Krylov subspace wave functions $\left|\mathcal{K}_{N}\right\rangle$ are determined.

To conclude, apart from $\left|\mathcal{K}_{0}\right\rangle$ and $\left|\mathcal{K}_{1}\right\rangle$, which correspond to an electron localized at site $i=0$ and site $i=1$ with zero phonon modes excited, respectively, all other vectors $\left|\mathcal{K}_{N}\right\rangle$ represent excitations of the many-body system (electron and phonon modes) that progressively spread away from the interface into the acceptor (see Fig. 4).
[1] G. Li, R. Zhu, and Y. Yang, Polymer solar cells, Nat. Photonics 6, 153 (2012).

[2] S. Günes, H. Neugebauer, and N. S. Sariciftci, Conjugated polymer-based organic solar cells, Chem. Rev. 107, 1324 (2007).

[3] L. Lu, T. Zheng, Q. Wu, A. M. Schneider, D. Zhao, and L. $\mathrm{Yu}$, Recent advances in bulk heterojunction polymer solar cells, Chem. Rev. 115, 12666 (2015).

[4] X.-Y. Zhu, Q. Yang, and M. Muntwiler, Charge-transfer excitons at organic semiconductor surfaces and interfaces, Acc. Chem. Res. 42, 1779 (2009).

[5] T. Drori, C.-X. Sheng, A. Ndobe, S. Singh, J. Holt, and Z. V. Vardeny, Below-Gap Excitation of $\pi$-Conjugated PolymerFullerene Blends: Implications for Bulk Organic Heterojunction Solar Cells, Phys. Rev. Lett. 101, 037401 (2008).

[6] M. Hallermann, S. Haneder, and E. Da Como, Chargetransfer states in conjugated polymer/fullerene blends: Belowgap weakly bound excitons for polymer photovoltaics, Appl. Phys. Lett. 93, 053307 (2008).

[7] H. Shirakawa, E. J. Louis, A. G. MacDiarmid, C. K. Chiang, and A. J. Heeger, Synthesis of electrically conducting organic polymers: Halogen derivatives of polyacetylene, $(\mathrm{CH})_{x}$, J. Chem. Soc., Chem. Commun., 578 (1977).
[8] D. L. Morel, A. K. Ghosh, T. Feng, E. L. Stogryn, P. E. Purwin, R. F. Shaw, and C. Fishman, High-efficiency organic solar cells, Appl. Phys. Lett. 32, 495 (1978).

[9] G. Grancini, M. Maiuri, D. Fazzi, A. Petrozza, H. J. Egelhaaf, D. Brida, G. Cerullo, and G. Lanzani, Hot exciton dissociation in polymer solar cells, Nat. Mater. 12, 29 (2013).

[10] A. E. Jailaubekov, A. P. Willard, J. R. Tritsch, W.-L. Chan, N. Sai, R. Gearba, L. G. Kaake, K. J. Williams, K. Leung, P. J. Rossky et al., Hot charge-transfer excitons set the time limit for charge separation at donor/acceptor interfaces in organic photovoltaics, Nat. Mater. 12, 66 (2013).

[11] S. Gélinas, A. Rao, A. Kumar, S. L. Smith, A. W. Chin, J. Clark, T. S. van der Poll, G. C. Bazan, and R. H. Friend, Ultrafast longrange charge separation in organic semiconductor photovoltaic diodes, Science 343, 512 (2014).

[12] A. M. Ballantyne, T. A. M. Ferenczi, M. Campoy-Quiles, T. M. Clarke, A. Maurano, K. H. Wong, W. Zhang, N. StingelinStutzmann, J.-S. Kim, D. D. Bradley et al., Understanding the influence of morphology on poly (3-hexylselenothiophene): PCBM solar cells, Macromolecules 43, 1169 (2010).

[13] H. Hoppe and N. S. Sariciftci, Organic solar cells: An overview, J. Mater. Res. 19, 1924 (2004). 
[14] G. D'Avino, Y. Olivier, L. Muccioli, and D. Beljonne, Do charges delocalize over multiple molecules in fullerene derivatives? J. Mater. Chem. C 4, 3747 (2016).

[15] N. R. Tummala, Z. Zheng, S. G. Aziz, V. Coropceanu, and J.L. Brédas, Static and dynamic energetic disorders in the $\mathrm{C}_{60}$, $\mathrm{PC}_{61} \mathrm{BM}, \mathrm{C}_{70}$, and $\mathrm{PC}_{71} \mathrm{BM}$ fullerenes, J. Phys. Chem. Lett. 6, 3657 (2015).

[16] F. Gao and O. Inganäs, Charge generation in polymer-fullerene bulk-heterojunction solar cells, Phys. Chem. Chem. Phys. 16, 20291 (2014).

[17] A. De Sio and C. Lienau, Vibronic coupling in organic semiconductors for photovoltaics, Phys. Chem. Chem. Phys. 19, 18813 (2017).

[18] S. M. Falke, C. A. Rozzi, D. Brida, M. Maiuri, M. Amato, E. Sommer, A. De Sio, A. Rubio, G. Cerullo, E. Molinari et al., Coherent ultrafast charge transfer in an organic photovoltaic blend, Science 344, 1001 (2014).

[19] Y. Song, S. N. Clafton, R. D. Pensack, T. W. Kee, and G. D. Scholes, Vibrational coherence probes the mechanism of ultrafast electron transfer in polymer-fullerene blends, Nat. Commun. 5, 4933 (2014).

[20] G. Wellein and H. Fehske, Polaron band formation in the Holstein model, Phys. Rev. B 56, 4513 (1997).

[21] G. Wellein and H. Fehske, Self-trapping problem of electrons or excitons in one dimension, Phys. Rev. B 58, 6208 (1998).

[22] H. De Raedt and A. Lagendijk, Numerical calculation of path integrals: The small-polaron model, Phys. Rev. B 27, 6097 (1983).

[23] P. E. Kornilovitch, Continuous-Time Quantum Monte Carlo Algorithm for the Lattice Polaron, Phys. Rev. Lett. 81, 5382 (1998).

[24] C. A. Rozzi, S. M. Falke, N. Spallanzani, A. Rubio, E. Molinari, D. Brida, M. Maiuri, G. Cerullo, H. Schramm, J. Christoffers et al., Quantum coherence controls the charge separation in a prototypical artificial light-harvesting system, Nat. Commun. 4, 1602 (2013).

[25] E. Jeckelmann and S. R. White, Density-matrix renormalization-group study of the polaron problem in the Holstein model, Phys. Rev. B 57, 6376 (1998).

[26] O. S. Barišić, Variational study of the Holstein polaron, Phys. Rev. B 65, 144301 (2002).

[27] M. Potthoff and W. Nolting, Surface metal-insulator transition in the Hubbard model, Phys. Rev. B 59, 2549 (1999).

[28] M. Potthoff and W. Nolting, Metallic surface of a Mott insulator-Mott insulating surface of a metal, Phys. Rev. B 60, 7834 (1999).

[29] M. Potthoff and W. Nolting, Dynamical mean-field study of the Mott transition in thin films, Eur. Phys. J. B 8, 555 (1999).

[30] P. Delange, T. Ayral, S. I. Simak, M. Ferrero, O. Parcollet, S. Biermann, and L. Pourovskii, Large effects of subtle electronic correlations on the energetics of vacancies in $\alpha$-Fe, Phys. Rev. B 94, 100102(R) (2016).

[31] S. Backes, T. C. Rödel, F. Fortuna, E. Frantzeskakis, P. LeFèvre, F. Bertran, M. Kobayashi, R. Yukawa, T. Mitsuhashi, M. Kitamura, K. Horiba, H. Kumigashira, R. Saint-Martin, A. Fouchet, B. Berini, Y. Dumont, A. J. Kim, F. Lechermann, H. O. Jeschke, M. J. Rozenberg, R. Valentí, and A. F. SantanderSyro, Hubbard band versus oxygen vacancy states in the correlated electron metal $\mathrm{SrVO}_{3}$, Phys. Rev. B 94, 241110(R) (2016).
[32] D. Jacob, K. Haule, and G. Kotliar, Dynamical mean-field theory for molecular electronics: Electronic structure and transport properties, Phys. Rev. B 82, 195115 (2010).

[33] V. Turkowski, A. Kabir, N. Nayyar, and T. S. Rahman, Dynamical mean-field theory for molecules and nanostructures, J. Chem. Phys. 136, 114108 (2012).

[34] S. Bera, N. Gheeraert, S. Fratini, S. Ciuchi, and S. Florens, Impact of quantized vibrations on the efficiency of interfacial charge separation in photovoltaic devices, Phys. Rev. B 91, 041107(R) (2015).

[35] S. Athanasopoulos, S. Tscheuschner, H. Bässler, and A. Köhler, Efficient charge separation of cold charge-transfer states in organic solar cells through incoherent hopping, J. Phys. Chem. Lett. 8, 2093 (2017).

[36] T. N. Aram, A. Asgari, and D. Mayou, Charge separation in organic solar cells: Effects of Coulomb interaction, recombination and hole propagation, Europhys. Lett. 115, 18003 (2016).

[37] R. Long and O. V. Prezhdo, Asymmetry in the electron and hole transfer at a polymer-carbon nanotube heterojunction, Nano Lett. 14, 3335 (2014).

[38] L.-C. Ku, S. A. Trugman, and J. Bonča, Dimensionality effects on the Holstein polaron, Phys. Rev. B 65, 174306 (2002).

[39] Y. Xie, J. Zheng, and Z. Lan, Full-dimensional multilayer multiconfigurational time-dependent Hartree study of electron transfer dynamics in the anthracene/C60 complex, J. Chem. Phys. 142, 084706 (2015).

[40] K. M. Pelzer and S. B. Darling, Charge generation in organic photovoltaics: A review of theory and computation, Mol. Syst. Des. Eng. 1, 10 (2016).

[41] B. A. Gregg, Entropy of charge separation in organic photovoltaic cells: The benefit of higher dimensionality, J. Phys. Chem. Lett. 2, 3013 (2011).

[42] P. W. Anderson, Absence of diffusion in certain random lattices, Phys. Rev. 109, 1492 (1958).

[43] R. Haydock and A. Mookerjee, Localization and its absence in CPA calculations, J. Phys. C 7, 3001 (1974).

[44] J. K. Freericks, S. Han, K. Mikelsons, and H. R. Krishnamurthy, Generalized gradient expansion for inhomogeneous dynamical mean-field theory: Application to ultracold atoms in a harmonic trap, Phys. Rev. A 94, 023614 (2016).

[45] R. Haydock, The recursive solution of the Schrodinger equation, Solid State Phys. 35, 215 (1980).

[46] K.-D. Richler, S. Fratini, S. Ciuchi, and D. Mayou, Inhomogeneous dynamical mean-field theory of the small polaron problem, J. Phys.: Condens. Matter 30, 465902 (2018).

[47] Z. Zheng, N. R. Tummala, Y.-T. Fu, V. Coropceanu, and J.-L. Brédas, Charge-transfer states in organic solar cells: Understanding the impact of polarization, delocalization, and disorder, ACS Appl. Mater. Interfaces 9, 18095 (2017).

[48] G. D’ Avino, L. Muccioli, F. Castet, C. Poelking, D. Andrienko, Z. G. Soos, J. Cornil, and D. Beljonne, Electrostatic phenomena in organic semiconductors: Fundamentals and implications for photovoltaics, J. Phys.: Condens. Matter 28, 433002 (2016).

[49] V. P. Antropov, O. Gunnarsson, and A. I. Liechtenstein, Phonons, electron-phonon, and electron-plasmon coupling in C60 compounds, Phys. Rev. B 48, 7651 (1993).

[50] C. Faber, J. L. Janssen, M. Côté, E. Runge, and X. Blase, Electron-phonon coupling in the $\mathrm{C}_{60}$ fullerene within the manybody GW approach, Phys. Rev. B 84, 155104 (2011). 
[51] F. Castet, G. D’Avino, L. Muccioli, J. Cornil, and D. Beljonne, Charge separation energetics at organic heterojunctions: On the role of structural and electrostatic disorder, Phys. Chem. Chem. Phys. 16, 20279 (2014).

[52] M. H. Gutknecht, A brief introduction to Krylov space methods for solving linear systems, in Frontiers of Computational Science, edited by Y. Kaneda, H. Kawamura, and M. Sasai (Springer, Berlin, Heidelberg, 2007), pp. 53-62.

[53] S. Ciuchi, F. de Pasquale, S. Fratini, and D. Feinberg, Dynamical mean-field theory of the small polaron, Phys. Rev. B 56, 4494 (1997).

[54] A. Liu, S. Zhao, S.-B. Rim, J. Wu, M. Könemann, P. Erk, and P. Peumans, Control of electric field strength and orientation at the donor-acceptor interface in organic solar cells, Adv. Mater. 20, 1065 (2008).
[55] C. Poelking and D. Andrienko, Design rules for organic donoracceptor heterojunctions: Pathway for charge splitting and detrapping, J. Am. Chem. Soc. 137, 6320 (2015).

[56] D. P. McMahon, D. L. Cheung, and A. Troisi, Why holes and electrons separate so well in polymer/fullerene photovoltaic cells, J. Phys. Chem. Lett. 2, 2737 (2011).

[57] C. Poelking, M. Tietze, C. Elschner, S. Olthof, D. Hertel, B. Baumeier, F. Würthner, K. Meerholz, K. Leo, and D. Andrienko, Impact of mesoscale order on open-circuit voltage in organic solar cells, Nat. Mater. 14, 434 (2015).

[58] A. Wilke, P. Amsalem, J. Frisch, B. Bröker, A. Vollmer, and N. Koch, Electric fields induced by energy level pinning at organic heterojunctions, Appl. Phys. Lett. 98, 123304 (2011).

[59] C. Lanczos, An iteration method for the solution of the eigenvalue problem of linear differential and integral operators, J. Res. Natl. Bur. Stand. B 45, 255 (1950). 